\title{
Simulating autonomous driving styles: Accelerations for three road profiles
}

\author{
Juffrizal Karjanto ${ }^{1,2}$, Nidzamuddin Md. Yusof ${ }^{1,2^{*}}$, Jacques Terken ${ }^{1}$, Frank Delbressine ${ }^{1}$, \\ Muhammad Zahir Hassan ${ }^{1,3}$ and Matthias Rauterberg ${ }^{1}$ \\ ${ }^{1}$ Department of Industrial Design, Eindhoven University of Technology, Eindhoven, The Netherlands \\ ${ }^{2}$ Faculty of Mechanical Engineering, Universiti Teknikal Malaysia Melaka, Melaka, Malaysia \\ ${ }^{3}$ Faculty of Engineering Technology, Universiti Teknikal Malaysia Melaka, Melaka, Malaysia
}

\begin{abstract}
This paper presents a new experimental approach to simulate projected autonomous driving styles based on the accelerations at three road profiles. This study was focused on the determination of ranges of accelerations in triaxial direction to simulate the autonomous driving experience. A special device, known as the Automatic Acceleration and Data controller (AUTOACcD), has been developed to guide the designated driver to accomplish the selected accelerations based on the road profiles and the intended driving styles namely assertive, defensive and light rail transit (LRT). Experimental investigations have been carried out at three different road profiles (junction, speed hump, and corner) with two designated drivers with five trials on each condition. A driving style with the accelerations of LRT has also been included in this study as it is significant to the present methodology because the autonomous car is predicted to accelerate like an LRT, in such a way that it enables the users to conduct activities such as working on a laptop, using personal devices or eating and drinking while travelling. The results demonstrated that 92 out of 110 trials of the intended accelerations for autonomous driving styles could be achieved and simulated on the real road by the designated drivers. The differences between the two designated drivers were negligible, and the rates of succeeding in realizing the intended accelerations were high. The present approach in simulating autonomous driving styles focusing on accelerations can be used as a tool for experimental setup involving autonomous driving experience and acceptance.
\end{abstract}

\section{Introduction}

In Malaysia, the number of road accidents is steadily increasing while deaths remain static Recent studies have shown that the development of autonomous vehicle (AV) is rapidly growing, and most drivers have already accepted Advance Driving Assistance System (ADAS), which is the foundation of the autonomous driving system [1], [2]. The automakers promise that the ultimate level of automation is a fully AV where human drivers/users will no longer have to deal with the operational and tactical levels in driving

\footnotetext{
*Corresponding author: n.yusof@tue.nl
} 
anymore [3]. Therefore, future AV users are only expected to decide where they want to go, select the destination and let the AV does the rest, from driving the car, selecting the best route, dealing with other road users and all the tasks that the human drivers used to do. In that sense, an AV is expected to behave in optimized manners that promote safety, reliability and comfort to the users. However, the acceptance of comfort may vary depending on users' perception and can be very subjective due to many factors especially driving styles [4]. Taubman-Ben-Ari et al. [5] suggested that driving styles vary across drivers and identified the most driving-specific factors into four categories; reckless and careless driving style, anxious driving style, angry and hostile driving style, and patient and careful driving style.

An AV or self-driving car contains thousands of sensors, and the way the vehicle is driven is strictly based on optimized logic (based on algorithms that analyse the inputs from sensors). In contrast, human drivers are driving based on their "targeted feeling" and heavily influenced by their own personality traits [6], [7]. Hence, this may lead to mismatched in term of driving styles preference for different types of the future AV users. The way an AV accelerates and decelerates at the intersection, handles a corner and circles a roundabout will be unfamiliar to the human drivers/users. Furthermore, since the AV drivers/users are no longer in command (no hands on the wheel, no feet on the pedals), the AV will probably accelerate and decelerate in the style that did not induce mental and physical discomfort to the human drivers/users in order to keep safety and comfort intact.

In order for an $\mathrm{AV}$ to be an optimized driver without sacrificing the comfort and enabling the activities other than driving for the users inside the AV, the AV is predicted and suggested to accelerate and decelerate slower than the typical human driven car and faster (or maybe the same) than the light rail transit (LRT) [8]. The LRT systems could be used as guideline such that the ways the system accelerates and decelerates in longitudinal and lateral direction are within the acceptable level of comfort for the passengers. Activities such as drinking and eating without spilling, and working or playing with electronic devices without experiencing physical discomfort like motion sickness as has been predicted would happen in the future AV riding experience [9], [10]. These activities were made possible since the induced forces felt by the users inside the LRT were minimized. In addition, the experience in riding an LRT is likely to be the benchmark used by the automotive players for their future development of AV since the LRT concept has been proven as one of the best options for the user to conduct secondary tasks while travelling in a moving vehicle [8]. On the same hand, future AV users who have had the experience travelling by train or LRT would draw mental scenarios of how traveling inside an AV should be as this new technology will also offer the kind of travelling without the need to drive.

A typical human driven car accelerates around $0.79 \mathrm{~m} / \mathrm{s}^{2}$ to $4.86 \mathrm{~m} / \mathrm{s}^{2}$ after leaving a junction or signalized intersection as stated in the work of Bogdanović and Ruškić [11]. They studied the various accelerations of cars, using digital cameras and programming, starting from the stop line at a juncture with traffic lights for ten intersections in one of the towns in Serbia. For the deceleration when approaching a junction or signalized intersection, El-Shawarby et al. [12] found that with 821 events, the range is between 1.51 $\mathrm{m} / \mathrm{s}^{2}$ to $7.47 \mathrm{~m} / \mathrm{s}^{2}$. The study was done with 60 participants in the enclosed area where it was safe to conduct the experiment. On the other hand, for the lateral accelerations, the ranges are influenced by the radius of the corner or roundabout on which the car is travelling [13]. Hugemann and Nickel (2003) [13] revealed that drivers usually produced smaller lateral accelerations with smaller radii corners while, in contrast, higher radii would usually associate with higher lateral accelerations. For the sharp turn or corner with radius in between 20 to 40 meters, the $10^{\text {th }}, 50^{\text {th }}$ and $90^{\text {th }}$ percentile of the lateral acceleration 
found is $3.1 \mathrm{~m} / \mathrm{s}^{2}, 4.1 \mathrm{~m} / \mathrm{s}^{2}$ and $5.3 \mathrm{~m} / \mathrm{s}^{2}$, respectively. Table 1 shows the list of accelerations in multi-directions for both the LRT and human driven car.

Table 1. Longitudinal and lateral accelerations and decelerations for light rail transit and human driven cars [11]-[15].

\begin{tabular}{|l|c|c|c|}
\hline & LRT & $\begin{array}{c}\text { Typical human- } \\
\text { driven car }\end{array}$ & $\begin{array}{c}\text { Upper limit in } \\
\text { human-driven car }\end{array}$ \\
\hline $\begin{array}{l}\text { Longitudinal } \\
\text { acceleration }\end{array}$ & $1.34 \mathrm{~m} / \mathrm{s}^{2}$ or $0.14 \mathrm{~g}$ & $2.47 \mathrm{~m} / \mathrm{s}^{2}$ or $0.25 \mathrm{~g}$ & $4.86 \mathrm{~m} / \mathrm{s}^{2}$ or $0.5 \mathrm{~g}$ \\
\hline $\begin{array}{l}\text { Longitudinal } \\
\text { deceleration }\end{array}$ & $-1.34 \mathrm{~m} / \mathrm{s}^{2}$ or $0.14 \mathrm{~g}$ & $-3.27 \mathrm{~m} / \mathrm{s}^{2}$ or $-0.33 \mathrm{~g}$ & $-7.47 \mathrm{~m} / \mathrm{s}^{2}$ or $-0.76 \mathrm{~g}$ \\
\hline $\begin{array}{l}\text { Lateral } \\
\text { acceleration }\end{array}$ & $1.47 \mathrm{~m} / \mathrm{s}^{2}$ or $0.15 \mathrm{~g}$ & $4.10 \mathrm{~m} / \mathrm{s}^{2}$ or $0.42 \mathrm{~g}$ & $5.30 \mathrm{~m} / \mathrm{s}^{2}$ or $0.54 \mathrm{~g}$ \\
\hline
\end{tabular}

For the vertical acceleration, Weber and Braaksma [16] stated that the measurement of discomfort, in terms of vertical force when traversing a hump, is depending on root sum of square (RSS) acceleration or peak vertical acceleration. The RSS value that indicates physical discomfort towards the motorist was at $0.17 \mathrm{~g}$, while peak vertical acceleration of $0.7 \mathrm{~g}$, is identified as heavily associated with human physical discomfort.

This paper reports the development and the execution of the experimental technique used in simulating the AV driving style in real road scenario that involves cornering, junction, and speed hump. A significant challenge to this research was to simulate the autonomous driving styles based on the selected accelerations at different road profiles. The present approach consists of experimental work that was carried out and validated using onvehicle site testing with two designated drivers who attempted to simulate the intended accelerations using a special device which is called as the Automatic Acceleration and Data controller (AUTOACCD).

The outcomes of this experiment were mapped with the results of calculated accelerations measurement from the data gained from the testing vehicle. In addition, the simulated data from the two designated drivers were tabulated and compared.

The current approach in simulating autonomous driving styles focusing on the accelerations in the triaxial direction can be used as a tool for experimental research on future autonomous driving experience and acceptance, especially in the preferred accelerations and comfort determination.

\section{Methodologies}

The methodology of the present study is shown in Fig. 1. The process started with the determination of driving styles to be simulated. Next, the process continued with the selection of road profiles that consist of a straight lane approaching and leaving a junction, a cornering, and a speed hump. Afterwards, the determination process of the driving styles to be simulated and followed by the determination of ranges of the accelerations for the selected driving styles based on the selected road profiles. Finally, the outcomes were examined in twofold analyses; first, was the comparison between the measured accelerations data using AUTOAccD and the calculated accelerations using the On-Board Diagnostic (OBD) data from the testing vehicle and second, was the comparison between accelerations simulated by the two designated drivers. 


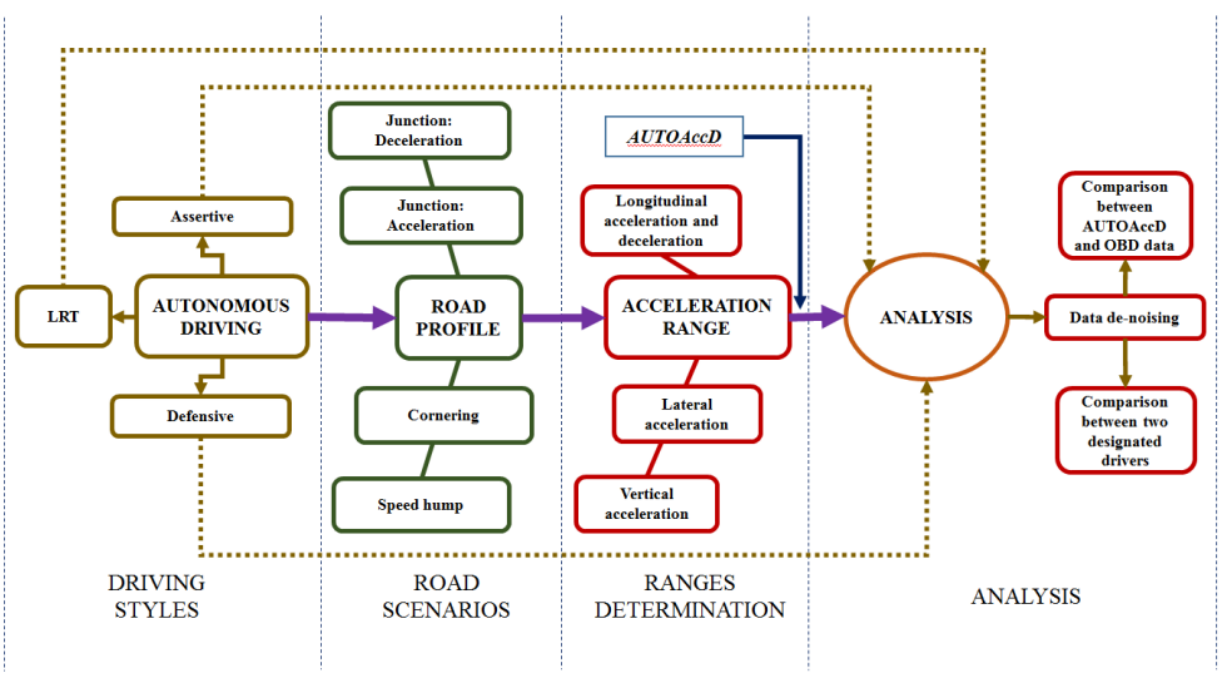

Fig. 1. The methodology of the present study.

\subsection{Driving style}

The first step was to determine the type of driving styles to be simulated. Elander et al. [4] defined driving style as the approach individuals select to manoeuvre their cars and something they accustom to over the years. In addition, driving styles include the selection of driving speeds in different circumstances, distance to others, the decision to overtake and tendency to violate traffic laws. Wang and Lukic [17] reviewed and summarized that driving styles are usually clustered into three categories; mild drivers (calm driving or economical driving style), normal drivers (medium driving style), and aggressive drivers (sporting driving style). Likewise, Taubman-Ben-Ari et al. [5] created the Multidimensional Driving Style Inventory (MSDI), which was modified from existing questionnaires (e.g., [18], [19]). Thus, driving styles vary across drivers and therefore more than one driving style for autonomous driving experience would be required [20]. In this study, three types of future autonomous driving styles of interest were selected which are defensive, assertive and LRT.

\subsection{Road scenario}

An Audi A3 was used as the testing vehicle for this experiment, provided by the Automotive Lab, Eindhoven University of Technology. The experimental investigation was carried out based on three different urban road profiles consisting of a junction (to access longitudinal accelerations and decelerations), speed hump (to access vertical accelerations) and corner (to access lateral accelerations). The route is located within the private compound of the Science Park campus, Eindhoven University of Technology, Eindhoven, Netherlands.

\subsection{Ranges of accelerations determination}

The ranges of lateral and longitudinal accelerations and deceleration to be implemented in order to simulate the LRT, defensive and assertive driving styles are presented in Fig. 2. These parameters are based on the data from LRT and typical human driven cars as 
reported in previous works [8], [11]-[13], [15]. For the longitudinal acceleration and deceleration, where the time plays a major role in getting the selected accelerations and decelerations, data for required time to generate the intended forces was calculated. In addition, the targeted velocity (the ending velocity in simulating acceleration or the starting velocity in simulating deceleration) would determine the required time in order to achieve the intended accelerations or decelerations and depending on where the experiment is done, the speed limit of that particular route needs to be taken into consideration.

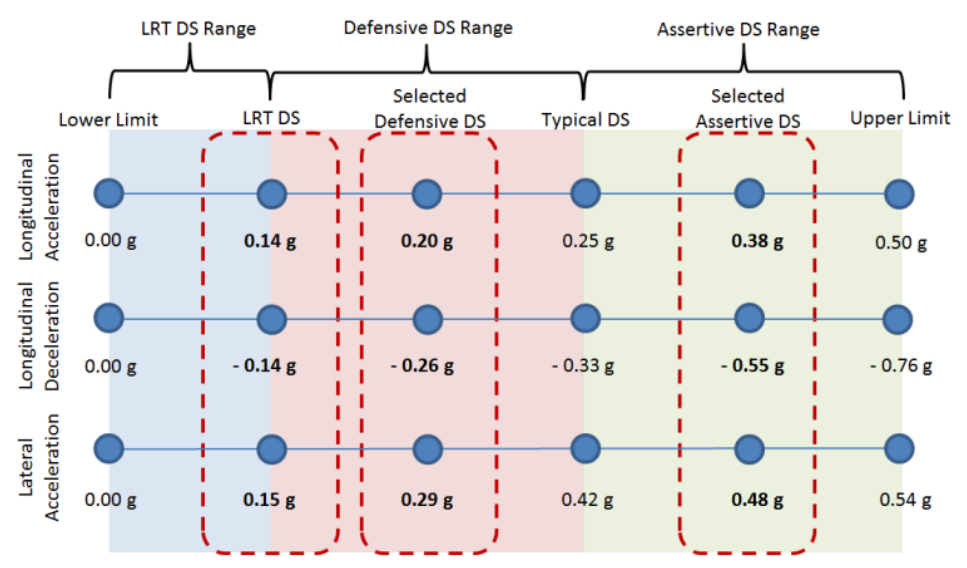

Fig. 2. The range of selection for defensive and assertive driving style for lateral and longitudinal accelerations and deceleration.

Next, the lateral acceleration, which is the centrifugal acceleration that pushes the vehicle to the outward of the curve during a cornering process, was determined. The centrifugal acceleration $\left(a_{c}\right)$ formulation is shown in Eq. 1 below:

$$
a_{c}=\frac{v^{2}}{r}
$$

where $v$ and $r$ represent travelling velocity in the corner and radius of the corner, respectively. In order to achieve the intended lateral accelerations as has been shown in Fig. 2, the selection of velocity has to be determined based on the parameters depicted in Table 2 below.

Table 2. Required velocity to generate lateral acceleration during cornering.

\begin{tabular}{|c|c|c|c|}
\hline \multirow{2}{*}{$\begin{array}{l}\text { Driving } \\
\text { style }\end{array}$} & \multirow{2}{*}{$\begin{array}{l}\text { Lateral acceleration } \\
\text { during cornering }\end{array}$} & \multicolumn{2}{|c|}{ Velocity } \\
\hline & & $(\mathrm{m} / \mathrm{s})$ & $(\mathbf{k m} / \mathbf{h})$ \\
\hline LRT & $0.15 \mathrm{~g}$ or $1.47 \mathrm{~m} / \mathrm{s}^{2}$ & 4.79 & 17.24 \\
\hline Defensive & $0.29 \mathrm{~g}$ or $2.84 \mathrm{~m} / \mathrm{s}^{2}$ & 6.66 & 23.97 \\
\hline Assertive & $0.48 \mathrm{~g}$ or $4.70 \mathrm{~m} / \mathrm{s}^{2}$ & 8.57 & 30.85 \\
\hline
\end{tabular}

As for the vertical acceleration, the selections of velocity when approaching the speed hump for different driving styles are shown in Fig. 3 and Table 3. 


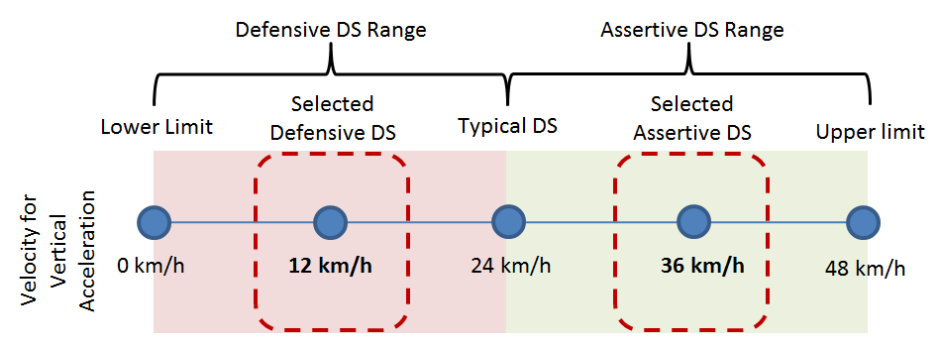

Fig. 3. The range of selection of velocity approaching the speed hump.

Table 3. Required velocity to generate vertical acceleration at the designated speed hump.

\begin{tabular}{|l|l|l|l|c|}
\hline \multirow{2}{*}{ Driving style } & \multicolumn{2}{|c|}{ Velocity } & \multicolumn{2}{c|}{$\begin{array}{c}\text { Vertical } \\
\text { acceleration }\end{array}$} \\
\cline { 2 - 5 } & \multicolumn{1}{|c|}{$\mathbf{( m / s )}$} & \multicolumn{1}{|c|}{$\mathbf{k m} / \mathbf{h})$} & $\mathbf{( m / \mathbf { s } ^ { 2 }}$ & $\mathbf{( g )}$ \\
\hline Defensive & $0-6.7$ & $0-24.0$ & 0.39 & 0.04 \\
\hline Assertive & $6.7-13.3$ & $24.0-48.0$ & 3.72 & 0.38 \\
\hline
\end{tabular}

Height of the speed hump $=0.10 \mathrm{~m}$

Length of the speed hump $=4.07 \mathrm{~m}$

\subsection{Automatic acceleration and data controller (AUTOAccD)}

A special dedicated device, the Automatic Acceleration and Data controller (AUTOAccD), was specifically developed with dual capabilities: to assist the designated driver to drive according to the defined acceleration condition and also act as instrumented processor to measure and collect the vehicle dynamics data. AUTOACCD consists of an OBD-II adapter that is connected to Arduino board and LCD display as shown in Fig. 4. OBD is an automotive term referring to a vehicle's self-diagnostic and is able to monitor real-time engine parameters such as speed, fuel consumption and transmission of a vehicle. The AUTOACCD was developed based on the data logger kit from Freematics [21] which comprised of Global Positioning System (GPS) receiver, triple-axis Micro-ElectroMechanical Systems (MEMS) accelerometers and gyro meters. In addition, the data logger was connected to a live display in the form of LCD screen. All the measured and collected data are stored in a micro Secure Digital (SD) card for analysis later.

The display was designed to assist the designated driver in maintaining a certain driving style by showing the specific ranges of lateral and longitudinal forces during the experiment. The display served as assistance and assurance device to the driver, apart from the training and his own experience in driving, in order to achieve the required acceleration at the specific location. There are three colours available to indicate the instantaneous driving style, in which LRT is indicated by purple colour while defensive and assertive are shown by green and red colours, respectively. In addition, there are two axes on which the vertical axis represents the longitudinal direction (upward movements indicates positive direction and vice versa) while the horizontal axis represents the lateral direction (rightward movements indicates positive direction and vice versa. 


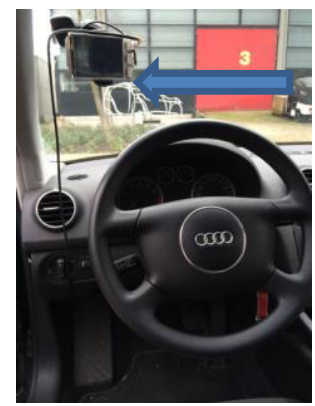

(a)

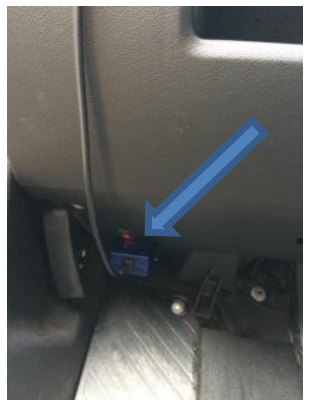

(b)

Fig. 4. (a) The position of the $A U T O A C C D$ in the car; and (b) the placement of the data logger and OBD.

\subsection{Data analysis}

Recent developments in Micro-Electro-Mechanical Systems (MEMS) technology have enabled the development of miniaturized sensors units like accelerometers and gyro meters. However, working with data obtained from the experiments involving measurements by these devices usually comes with noise ranging from alignment errors to random noise. Hence, the noise has to be filtered for further data analysis and interpretation. Rather than using the traditional filters for de-noising such as time-frequency domain filters like Fourier transform, a time-scale domain filter known as Wavelet transform was employed. The Wavelet de-noising method was implemented because its ability to remove noises and retain the signal regardless the frequency of the signal [22], [23]. For this study, the Wavelet de-noising was done using Matlab (Wavemenu) and the selection of the appropriate filter was based on the previous literature using the same equipment. The Daubechies filter level three ( $\mathrm{Db} 3$ ) was used in order to remove the high-frequency noise from the MEMS accelerometers [24].

After the de-noising process, the acceleration data from the two designated drivers were compared to check the ability of the AUTOAccD in assisting the drivers to simulate the intended accelerations. In addition, the accuracies and consistencies of the simulated acceleration will also be discussed. Next, the comparison between the acceleration data gained from the AUTOAccD and the calculated data from the testing vehicle's OBD was done to check the discrepancies between the two tools.

\section{Results and discussion}

The analyses started with the process of filtering or de-noising of the signal attained from the accelerometers. Wavemenu function in Matlab was used for its simplicity and its elegant visual tools that enable the users to interactively de-noise a signal and instantaneously display the result on the screen [25]. After the de-noising process, the acceleration data were analysed in terms of simulations from the two designated drivers and were compared with the calculated data that were gained from the vehicle's system.

\subsection{Comparison between data measured by AUTOAccD and calculated from OBD}

The comparison between acceleration data (longitudinal and lateral) gained from the triaxial accelerometers within the AUTOAccD and calculated using the speed extracted 
from the vehicle's OBD is shown in Table 4. For the longitudinal accelerations and decelerations, the largest variances were found in the simulation of assertive driving style where as much as $0.06 \mathrm{~g}$ and $0.12 \mathrm{~g}$ differences were recorded, respectively. Otherwise, for the defensive and LRT driving style, the differences in means between AUTOAccD and OBD were between $0.00 \mathrm{~g}$ to $0.03 \mathrm{~g}$, which indicated higher accuracies. Meanwhile, for the lateral acceleration, between the three driving styles, AUTOAccD for assertive driving style yielded the highest difference with $0.14 \mathrm{~g}$ while the defensive driving style registered a difference of $0.05 \mathrm{~g}$ and an LRT driving style with the smallest difference with $0.01 \mathrm{~g}$.

Table 5 shows the comparison between vertical acceleration data collected from the AUTOAccD and determined from the vehicle's OBD. The differences in means for each of the driving styles were $0.03 \mathrm{~g}$ and $0.04 \mathrm{~g}$. In addition, the standard deviations recorded were between 0.00 and 0.06 which showed great consistencies among the two set of data. From Table 4 and Table 5, similar patterns can be observed in which all the measured acceleration data were higher than the calculated ones. These phenomena were parallel with the previous work of Brol and Mamala [26] who found comparable results with the measured data having the differences up to $0.10 \mathrm{~g}$. The differences were contributed from the road irregularities, and some were from the engine noises. The road surface irregularities, in this study, were the cobblestones road that generated additional accelerations through the vehicle suspension system and was recorded by the accelerometers which were attached to the vehicle's body.

Table 4. Comparison between longitudinal and lateral accelerations data measured by AUTOAccD and calculated from OBD.

\begin{tabular}{|c|c|c|c|c|c|c|c|}
\hline \multicolumn{2}{|c|}{ Type of acceleration } & \multicolumn{2}{|c|}{ Assertive DS } & \multicolumn{2}{|c|}{ Defensive DS } & \multicolumn{2}{|c|}{ LRT DS } \\
\hline & Mean & $\begin{array}{c}\begin{array}{c}\text { AUTO } \\
\text { AccD }\end{array} \\
0.41\end{array}$ & $\begin{array}{c}\text { OBD } \\
0.35 \\
\end{array}$ & $\begin{array}{c}\begin{array}{c}\text { AUTO } \\
\text { AccD }\end{array} \\
0.22 \\
\end{array}$ & $\begin{array}{c}\text { OBD } \\
0.21 \\
\end{array}$ & $\begin{array}{c}\begin{array}{c}\text { AUTO } \\
\text { AccD }\end{array} \\
0.09 \\
\end{array}$ & $\begin{array}{c}\text { OBD } \\
0.07\end{array}$ \\
\hline acceleration & SD & 0.07 & 0.06 & 0.06 & 0.06 & 0.04 & 0.05 \\
\hline \multirow{2}{*}{$\begin{array}{l}\text { Longitudinal } \\
\text { deceleration }\end{array}$} & Mean & -0.48 & -0.36 & -0.32 & -0.29 & -0.15 & -0.15 \\
\hline & SD & 0.07 & 0.10 & 0.04 & 0.09 & 0.03 & 0.03 \\
\hline \multirow{2}{*}{$\begin{array}{l}\text { Lateral } \\
\text { acceleration }\end{array}$} & Mean & 0.54 & 0.39 & 0.31 & 0.26 & 0.12 & 0.11 \\
\hline & SD & 0.12 & 0.05 & 0.03 & 0.02 & 0.04 & 0.03 \\
\hline
\end{tabular}

Table 5. Comparison between vertical accelerations data measured by AUTOAccD and calculated from OBD.

\begin{tabular}{|l|c|c|c|c|}
\hline \multirow{2}{*}{$\begin{array}{c}\text { Vertical } \\
\text { acceleration }\end{array}$} & \multicolumn{2}{|c|}{ Assertive DS } & \multicolumn{2}{c|}{ Defensive DS } \\
\cline { 2 - 5 } & $\begin{array}{c}\text { AUTO } \\
\text { AccD }\end{array}$ & OBD & $\begin{array}{c}\text { AUTO } \\
\text { AccD }\end{array}$ & OBD \\
\hline Mean & 0.33 & 0.30 & 0.07 & 0.03 \\
\hline SD & 0.06 & 0.01 & 0.01 & 0.00 \\
\hline
\end{tabular}

\subsection{Comparison between two designated drivers}

For the leaving-a-junction scenario, the tabulated accelerations simulated by the two designated drivers using three driving styles (with five trials for each driving style) are presented in Fig. 5. Additionally, the means and standard deviations (SD) are presented in Table 6 . For the simulated accelerations by using LRT driving style, both drivers managed to simulate the accelerations within the desired range, although the means of the drivers were quite far apart, the SD indicated that they were very consistent in simulating this driving style. For the defensive and assertive driving styles, both of the drivers had fluctuated accelerations and were less consistent compared to when simulating the LRT driving style. Nonetheless, the means for both drivers in simulating the last two driving 
styles were closer to the intended accelerations. For the defensive driving style, out of ten trials altogether, three of the accelerations fell outside of the desired range, whereas for the assertive driving style, all the simulated accelerations were within the intended range.

In this particular scenario, the testing car was accelerating in one smooth motion where there was no jerking or any other traffic that would impede the acceleration process. In addition, in order to successfully simulate larger acceleration forces especially for the assertive driving style, the accelerations must be done in preferably with lower transmission such as the first gear. Higher acceleration forces were usually achieved with lower gears especially when the vehicle started to accelerate from a completely static position. Higher gears usually produce acceleration not as large as the acceleration produced from the lower gears after leaving an intersection as has been shown in the previous study by Bogdanović and Ruškić [11].

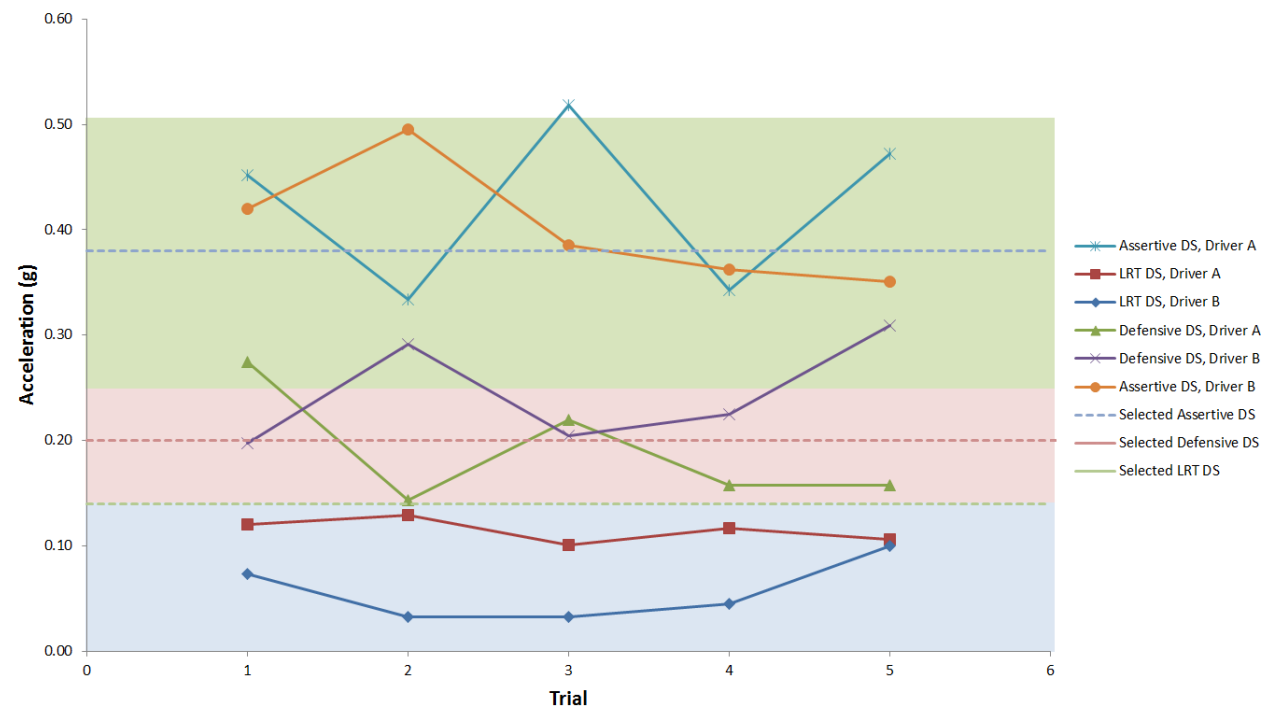

Fig. 5. Tabulated longitudinal accelerations for two designated drivers simulated with three driving styles.

Table 6. Means and SD of the three simulated driving styles for longitudinal accelerations for each driver, $\mathrm{n}_{\text {trial }}=5$.

\begin{tabular}{|c|c|c|c|c|c|c|}
\hline \multirow[b]{2}{*}{ Driver } & \multicolumn{2}{|c|}{$\begin{array}{c}\text { LRT DS } \\
(\text { Targeted acc. }=0.14 \mathrm{~g})\end{array}$} & \multicolumn{2}{|c|}{$\begin{array}{c}\text { Defensive DS } \\
(\text { Targeted acc. }=0.20 \mathrm{~g})\end{array}$} & \multicolumn{2}{|c|}{$\begin{array}{c}\text { Assertive DS } \\
\text { (Targeted acc. }=0.38 \mathrm{~g})\end{array}$} \\
\hline & Mean (g) & SD & Mean (g) & SD & Mean (g) & SD \\
\hline $\mathbf{A}$ & 0.11 & 0.01 & 0.19 & 0.06 & 0.42 & 0.08 \\
\hline B & 0.06 & 0.03 & 0.25 & 0.05 & 0.40 & 0.06 \\
\hline
\end{tabular}

For the straight lane approaching the junction scenario, the decelerations simulated by the two designated drivers using three driving styles, with five trials each, are tabulated in Fig. 6. In addition, the means and SDs are also presented in Table 7. For this scenario, both drivers showed high consistency in simulating the accelerations although driver B actually did not manage to register any acceleration within the desired LRT range, whereas, for the defensive and assertive driving style, much higher variances were produced by both of the drivers. In addition, the attempted decelerations generally deviated quite far from the intended means. For the assertive driving style, all the simulated decelerations were within the intended range, while for the defensive driving style, both of the drivers registered half of the trials outside the intended range. 
In simulating the deceleration, the drivers were instructed to decelerate, from a designated speed of $30 \mathrm{~km} / \mathrm{h}$, by adjusting the braking force according to the guides as shown on the AUTOAccD. However, in general, the simulations of deceleration are harder to generate compared to the simulations of acceleration. One of the reasons was that the drivers had to control both the clutch and brake pedal in order to achieve the designated deceleration rate and making sure the engine would not stall whereas, in the accelerations, the drivers only need to control the accelerator or gas throttle.

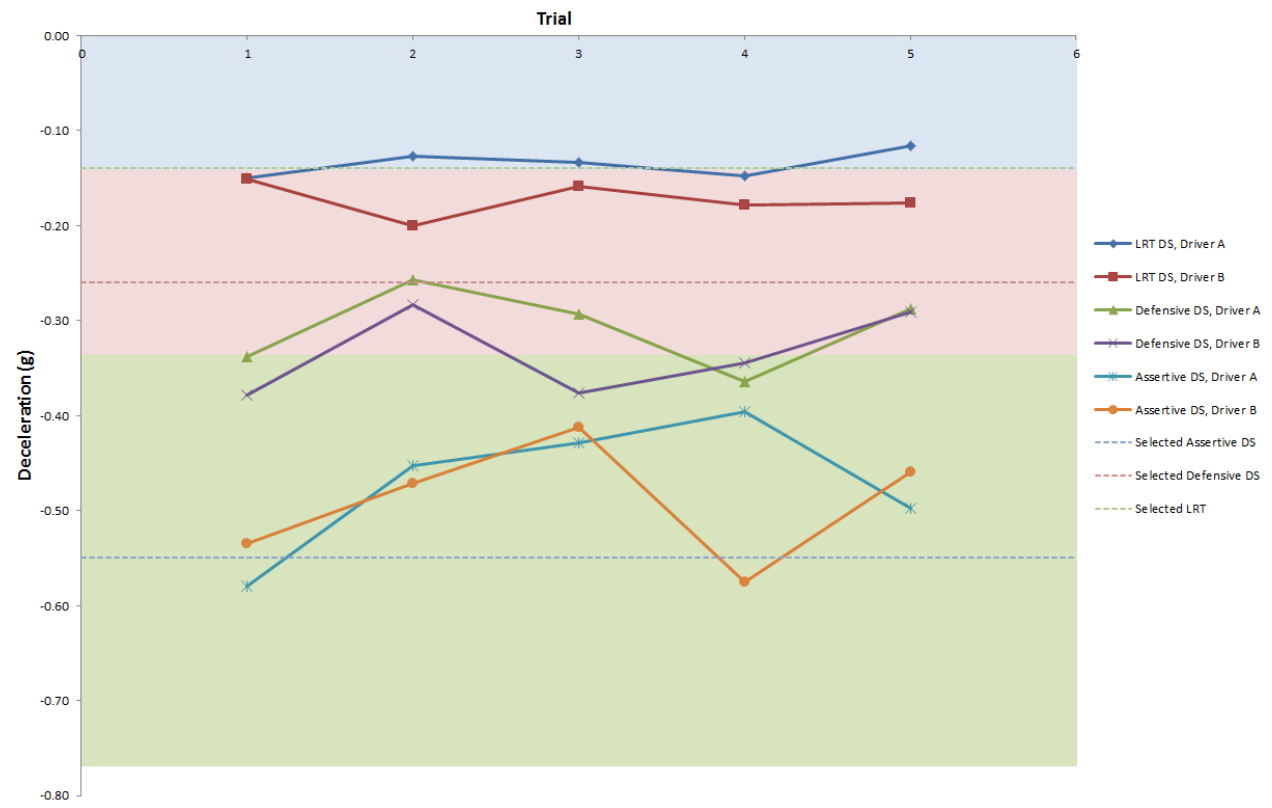

Fig. 6. Tabulated decelerations for two designated drivers simulated with three driving styles.

Table 7. Means and standard deviations of the three simulated driving styles for decelerations for each driver, $\mathrm{n}_{\text {trial }}=5$.

\begin{tabular}{|c|c|c|c|c|c|c|}
\hline \multirow{2}{*}{ Driver } & \multicolumn{2}{|c|}{$\begin{array}{c}\text { LRT DS } \\
\text { (Targeted acc. }=- \\
\text { 0.14g) }\end{array}$} & \multicolumn{2}{c|}{$\begin{array}{c}\text { Defensive DS } \\
\text { (Targeted acc. = - 0.26g) }\end{array}$} & \multicolumn{2}{c|}{$\begin{array}{c}\text { Assertive DS } \\
\text { (Targeted acc. = - 0.55g) }\end{array}$} \\
\cline { 2 - 7 } & Mean (g) & SD & Mean (g) & SD & Mean (g) & SD \\
\hline A & -0.13 & 0.01 & -0.31 & 0.04 & -0.47 & 0.07 \\
\hline B & -0.17 & 0.02 & -0.33 & 0.05 & -0.49 & 0.06 \\
\hline
\end{tabular}

For the cornering, the lateral accelerations simulated by the two designated drivers using three driving styles are presented in Fig. 7. In addition, the means and SDs are also presented in Table 8 . In the simulation of the LRT driving style, driver B had better consistencies compared to driver A as lower SD was registered. For the defensive driving style, in which both drivers achieved mean values that are higher than the intended one, both of the drivers registered good consistencies with SD of 0.03 each. Whereas for the simulation of assertive driving style, there were fluctuations in the simulated accelerations, as in this driving style the drivers had to generate accelerations that are above the average of typical human driving style. However, out of the combined ten trials from the two drivers, only once a simulated acceleration fell outside the lower limit of the intended assertive range. 
In simulating the lateral accelerations, it was crucial that the radius of the cornering was maintained to be as constant as possible. By referring back to Eq. (1), it is clear that both velocity and radius of the corner are playing a big role in determining the induced lateral acceleration [27]. In this study, the roads which have been used do not have any road markings on the surface, and therefore, both of the drivers would try to undertake the cornering by making sure the car was always approximately travelling in the middle of the road. As mentioned by Charlton [28], for more accurate results in cornering, a clearer and more accurate guideline or delineation treatment should be developed such as line marking on the road surface where the drivers can align themselves to make sure that for each trial, the radius of the travelling car in the corner stays almost the same every single time.

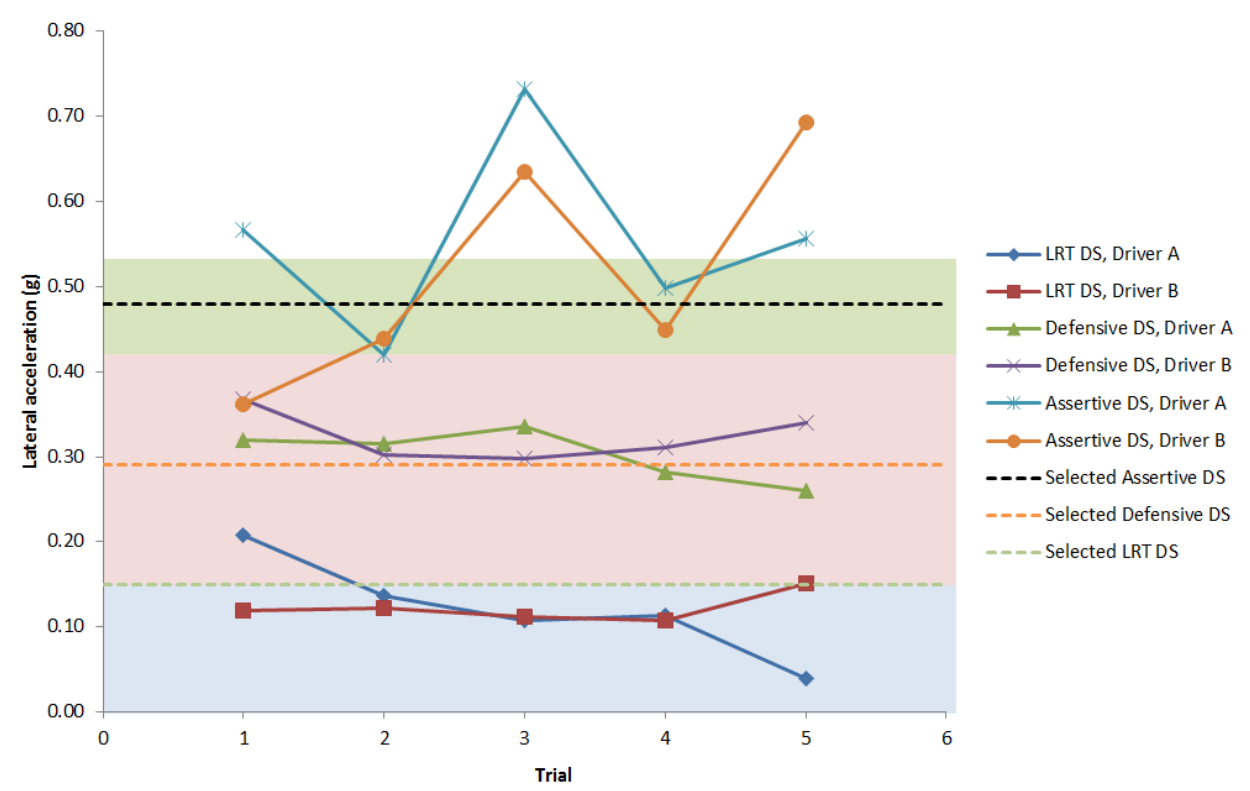

Fig. 7. Tabulated lateral accelerations for two designated drivers simulated with three driving styles.

Table 8. Means and standard deviations of the three simulated driving styles for lateral accelerations for each driver, $\mathrm{n}_{\text {trial }}=5$.

\begin{tabular}{|c|c|c|c|c|c|c|}
\hline \multirow{2}{*}{ Driver } & \multicolumn{2}{|c|}{ LRT DS } & \multicolumn{2}{c|}{$\begin{array}{c}\text { Defensive DS } \\
\text { (Targeted acc. }=0.15 g)\end{array}$} & \multicolumn{2}{c|}{$\begin{array}{c}\text { Assertive DS } \\
\text { (Targeted acc. = 0.29g) }\end{array}$} \\
\cline { 2 - 7 } & Mean (g) & SD & Mean (g) & SD & Mean (g) & SD \\
\hline A & 0.12 & 0.06 & 0.30 & 0.03 & 0.47 & 0.11 \\
\hline B & 0.12 & 0.02 & 0.32 & 0.03 & 0.46 & 0.14 \\
\hline
\end{tabular}

For the vertical acceleration, the simulated accelerations are presented in Fig. 8. In addition, the means and SDs are presented in Table 9. For this study, both designated drivers realized higher vertical acceleration means than the intended for the simulation of defensive driving style while for the assertive driving style, they realized lower means than the intended one. In term of SDs, for the defensive driving style, both drivers managed to simulate higher consistencies as indicated by low deviation values although for the Driver A, his accelerations were sometimes lower and some other time were higher than the intended means whereas, for driver B, all the recorded values were lower than the intended means. For the assertive driving style, driver A indicated lower consistencies compared to driver $\mathrm{B}$ as the SD recorded were 0.08 and 0.03 , respectively. From 
, all of the 20 trials from both drivers were recorded within the intended ranges of interest.

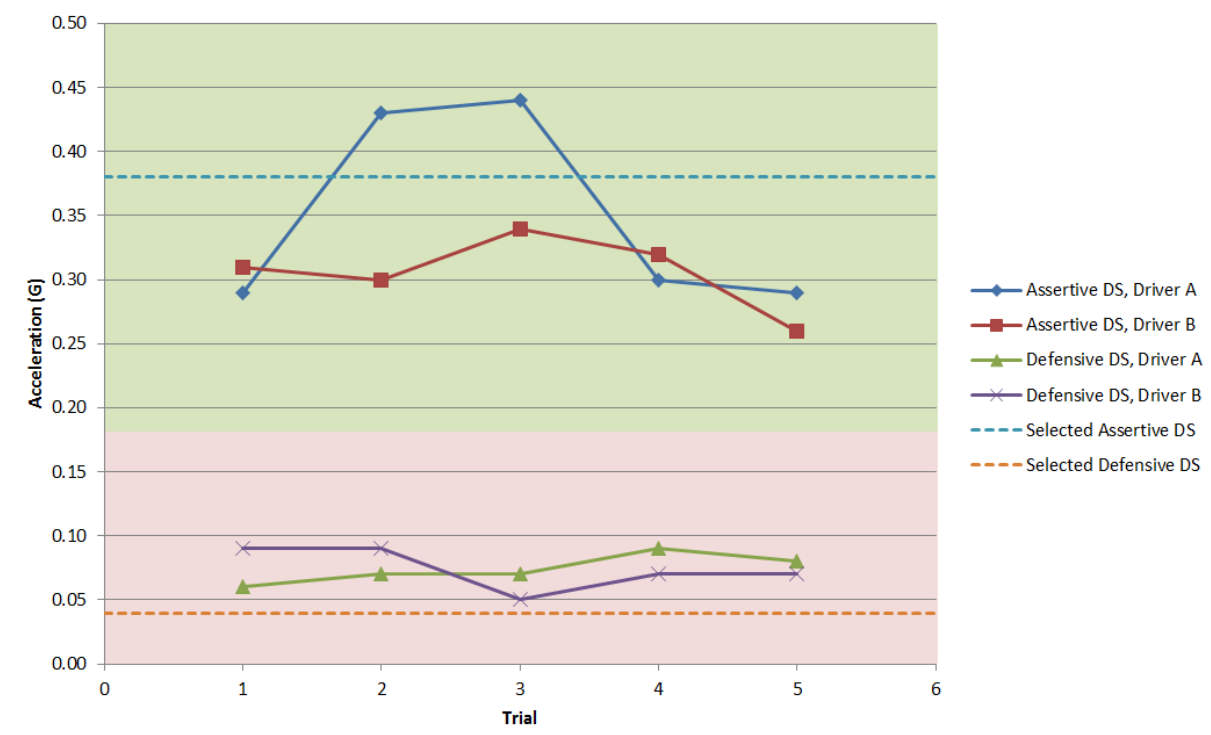

Fig. 8. Tabulated vertical accelerations for two designated drivers simulated with two driving styles.

Table 9. Means and standard deviations of the two simulated driving styles for vertical accelerations for each driver, $\mathrm{n}_{\text {trial }}=5$.

\begin{tabular}{|c|c|c|c|c|}
\hline \multirow{2}{*}{ Driver } & \multicolumn{2}{|c|}{$\begin{array}{c}\text { Defensive DS } \\
\text { (Targeted acc. }=\mathbf{0 . 0 4 g})\end{array}$} & \multicolumn{2}{c|}{$\begin{array}{c}\text { Assertive DS } \\
\text { (Targeted acc. }=\mathbf{0 . 3 8 g} \text { ) }\end{array}$} \\
\cline { 2 - 5 } & Mean (g) & SD & Mean (g) & SD \\
\hline A & 0.07 & 0.01 & 0.35 & 0.08 \\
\hline B & 0.07 & 0.02 & 0.31 & 0.03 \\
\hline
\end{tabular}

In simulating the vertical accelerations, the only parameter to be controlled was the approaching velocity. The main difference between a speed hump and a speed bump is that the former is designed for a vehicle to traverse at a low speed $(25-30 \mathrm{~km} / \mathrm{h})$ without the need to slow down while the latter require a vehicle to decelerate up to $8 \mathrm{~km} / \mathrm{h}$ when arriving at the tip of the bump [29]. Therefore, in this study, the intended assertive driving style required the drivers to traverse at the speed hump at a velocity of $36 \mathrm{~km} / \mathrm{h}$ which is relatively high for drivers with defensive or normal driving style. In addition, the available speed hump for this experiment was relatively short at $4.07 \mathrm{~m}$ in length whereas a good speed hump's length is usually around $6.70 \mathrm{~m}$ or even extending to $9.50 \mathrm{~m}$ in some countries like Denmark and $12.0 \mathrm{~m}$ in Australia [16]. A longer speed hump would induce peak acceleration at a higher speed as usually a car has a wheelbase with a similar length to the speed hump whereas a speed bump usually has a significantly shorter length compared to a car's wheelbase. Therefore, a speed bump would generate high vertical force even at a low speed and, therefore, would definitely induce discomfort if the experiment were done using the same conditions as in this study. Since only the velocity parameter was considered in the current study, the intended accelerations were lower than measured in defensive driving style simulation because the suspension effects were not included in the initial calculations. As highlighted by Shirahatti [30], due to the vehicle suspension, when the vehicle speed was increasing, the vertical acceleration was first increasing and then decreasing at a high rate before stabilized at around $25 \mathrm{~km} / \mathrm{h}$. The study also indicated that 
the vehicle's vertical displacement and pitch rotation were higher at low speed and came to be lower at high speed, similar to the finding in the current study in simulating assertive driving style.

\subsection{General discussion}

The simulation of autonomous driving styles at three road profiles namely straight lane approaching and leaving a junction, cornering, and speed hump could be done with the help of a special device known as AUTOAccD. The experiment set up started with the determination of interested driving styles and then followed with the selection of routes and determination of ranges for acceleration in different directions. In this study, the determination of driving styles was influenced by the intention to simulate autonomous driving experience with different driving styles. Therefore, three types of driving styles were determined, namely assertive, defensive and LRT driving style. The accelerations of LRT was exclusively included as riding in an LRT could already be a mental benchmark for future AV users as the experience of using an LRT was the closest one would get in imagining using an AV. This is in line with the public opinion regarding AV reported by Schoettle and Sivak in 2014 [31], who found that most of their questionnaire respondents would like to socialize with friends and family, do work-related activities, sleeping or resting, reading, or watching television or movies inside a future AV.

In this experiment set up, the implementation of AUTOAccD resulted in two main benefits. First, this device manages to help the drivers in simulating the intended accelerations by interactively showing and clustering the live acceleration data so that the drivers could adjust the throttle position and speed in order to get the required simulations. Second, the device also acts as a data logger that measure and stores various data for further analysis. Although the AUTOAccD would help the designated drivers in simulating the intended accelerations, practise is still required as it would help the drivers to get the feeling on how to generate higher accuracy simulations with fewer deviations.

In terms of comparison between the designated drivers, most of the acceleration data have small SDs and close to the intended means. In addition, both drivers managed to simulate accelerations within the intended ranges as out of 110 trials altogether only 18 values were simulated outside the intended ranges. By comparing the three driving styles, the assertive driving styles were the hardest to be simulated regardless of the road profiles. It is evidenced by the higher SD values for assertive driving styles compare to the other two driving styles. For the defensive driving styles, in general, although low deviations were recorded the average means for the simulated accelerations are usually higher than the intended ones. For the LRT driving styles, both means and SDs for the simulated accelerations indicated variations between the two drivers. Unlike the other two driving styles which distinguished patterns were shown, for the LRT driving styles both of the drivers did not show any clear trend regarding the simulated accelerations.

A few cautions have to be taken before executing the experiment on the road such as for the lateral accelerations where the cornering radius has to be maintained in order to generate the specified centrifugal forces. For the longitudinal accelerations, a lower gear preferably the first gear is recommended in order to achieve the intended accelerations. For the longitudinal deceleration, although a specific starting velocity has been identified, the simulation of deceleration required an act of balance between clutch and brake pedal and skill to produce a continuous and smooth deceleration. On the other hand, the process of analysing the accelerometers data began with the de-noising process in order to eliminate the noises without flattening the valuable data, from the three accelerometers in the tri-axial direction. 


\section{Conclusions}

In conclusion, this study of simulating AV driving styles could be extended with the evaluation of acceptance and preference of future autonomous driving styles such as using the subjective measurements (unipolar or bipolar comfort rating) and objective measurements (continuous physiological measures for example the galvanic skin conductance (GSR) and electrocardiogram (EKG)) [32]. The significant finding of the present work could be further extended in the area of comfort study in future AV driving styles and the effects of motion sickness where the role of the driver would change from the sole controller of the vehicle to a passive user with a much-minimized role. In addition, comfort in travelling is usually associated with the way the vehicle is controlled and driven. In the near future, where accelerations are controlled by the algorithms and machines, autonomous vehicle users who used to be the drivers may feel discomfort unless the driving styles of the vehicle are somewhat similar to their own. Hence, there is a special need to study the associations between simulated accelerations and driving styles in order to enhance the autonomous driving experience for the future AV users.

If such experiments were to be extended, experts from different backgrounds, like engineering and social sciences among others, should work together in order to solve these projected issues effectively and efficiently. The rising of AV technology brings along the benefits such as lowering the fatal accidents by taking the human out of the driving's loop and maximizing the traffic flow and usage of the road [33]. At the same time, studies of human-machine interaction and acceptance should be explored extensively because as marvellous as AV technology could be, humans are still the end users who will buy, use and experience the AV in the coming future.

The authors would like to express their gratitude and special acknowledgements to Universiti Teknikal Malaysia Melaka (UTeM) and Ministry of Education, Malaysia (KPM) for the funding of the Ph.D. programs of Juffrizal Karjanto and Nidzamuddin Md. Yusof and also the post-doctoral program of Muhammad Zahir Hassan in the Industrial Design Department, Eindhoven University of Technology, The Netherlands.

\section{References}

1. KPMG, "Self-Driving Cars: Are We Ready?," (2015)

2. KPMG, "Self-Driving Cars : The Next Revolution," (2012)

3. T. M. Gasser and D. Westhoff, "BASt-study: Definitions of Automation and Legal Issues in Germany," (2012)

4. J. Elander, R. West, and D. French, "Behavioral correlates of individual differences in road-traffic crash risk: An examination method and findings," Psychol. Bull., vol. 113, no. 2, pp. 279-294, (1993)

5. O. Taubman-Ben-Ari, M. Mikulincer, and O. Gillath, "The multidimensional driving style inventory - Scale construct and validation," Accid. Anal. Prev., vol. 36, no. 3, pp. 323-332, (2004)

6. T. Vaa, "From Gibson and Crooks to Damasio: The role of psychology in the development of driver behaviour models," Transp. Res. Part F Traffic Psychol. Behav., vol. 25, pp. 112-119, (2014)

7. H. Summala, "Towards understanding motivational and emotional factors in driver behaviour: Comfort through satisficing," in Modelling Driver Behaviour in Automotive Environments: Critical Issues in Driver Interactions with Intelligent Transport Systems, (2007), pp. 189-207

8. S. Le Vine, A. Zolfaghari, and J. Polak, "Autonomous cars: The tension between 
occupant experience and intersection capacity," Transp. Res. Part C, vol. 52, pp. 1-14, (2015)

9. C. Diels and J. E. Bos, "Self-driving carsickness," Appl. Ergon., vol. 53, pp. 374-382, (2016)

10. M. Sivak and B. Schoettle, "Motion Sickness in Self-Driving Vehicles (Report No. UMTRI-2015-12)," (2015)

11. V. Bogdanović and N. Ruškić, "The research of vehicle acceleration at signalized intersections," Traffic Transp., vol. 25, no. 1, pp. 33-42, (2013)

12. I. El-Shawarby, H. Rakha, V. Inman, and G. Davis, "Evaluation of Driver Deceleration Behavior at Signalized Intersections," Transp. Res. Rec. J. Transp. Res. Board, pp. 29$35,(2007)$

13. D. Hugemann and D. Nickel, "Longitudinal and Lateral Accelerations in Normal Day Driving," in 6th International Conference of The Institute of Traffic Accident Investigators, (2003)

14. Parsons Brinckerhoff Team, "California High Speed Train Program Environmental Impact Report/Environmental Impact Statement: Engineering Criteria," (2004)

15. TCRP, TCRP Report 155 - Track Design Handbook for Light Rail Transit, Second Edition. (2012)

16. P. A. Weber and J. P. Braaksma, "Towards a North American geometric design standard for speed humps," ITE J. (Institute Transp. Eng., vol. 70, no. 1, pp. 30-34, (2000)

17. R. Wang and S. M. Lukic, "Review of driving conditions prediction and driving style recognition based control algorithms for hybrid electric vehicles," 2011 IEEE Veh. Power Propuls. Conf. VPPC 2011, (2011)

18. E. Gulian, G. Matthews, A. I. Glendon, D. R. Davies, and L. M. Debney, "Dimensions of driver stress," Ergonomics, vol. 32, no. 6, pp. 585-602, (1989)

19. J. Reason, A. Manstead, S. Stradling, J. Baxter, and K. Campbell, "Errors and violations on the roads: a real distinction?.pdf," Ergonomics, vol. 33, no. 10/11, pp. 1315-1332, (1990)

20. M. Kuderer, S. Gulati, and W. Burgard, "Learning Driving Styles for Autonomous Vehicles from Demonstration," 2015 IEEE Int. Conf. Robot. Autom., vol. 134, pp. 2641-2646, (2015)

21. S. Huang, "OBD-II Telematics Advanced Kit (Arduino MEGA 2560)," (2014) [Online]. Available: http://freematics.com/pages/products/arduino-telematics-kit-3/.

22. C. Taswell, "The what, how, and why of wavelet shrinkage denoising," Comput. Sci. Eng., vol. 2, no. 3, pp. 1-11, (2000)

23. C. W. Kang, C. H. Kang, and C. G. Park, "Wavelet Denoising Technique for Improving of the Low Cost MEMS-GPS Integrated Navigation Sytems," Inetrnational Symp. GPS/GNSS, pp. 3-8, (2010)

24. A. M. Hasan, K. Samsudin, A. R. Ramli, and R. S. Azmir, "Wavelet-based pre-filtering for low cost inertial sensors," J. Appl. Sci., vol. 10, no. 19, pp. 2217-2230, (2010)

25. M. Misiti, Y. Misiti, G. Oppenheim, and J.-M. Poggi, Wavelet toolbox. (1996)

26. S. Brol and J. Mamala, "Assessment of passenger car driveability with use of two axis accelerometer mounted on car body," J. Ultragarsas, vol. 2, no. 2, pp. 31-36, (2006)

27. G. Reymond, a Kemeny, J. Droulez, and a Berthoz, "Role of lateral acceleration in curve driving: driver model and experiments on a real vehicle and a driving simulator.," Hum. Factors, vol. 43, no. 3, pp. 483-495, (2001)

28. S. G. Charlton, "The role of attention in horizontal curves: A comparison of advance warning, delineation, and road marking treatments," Accid. Anal. Prev., vol. 39, no. 5, pp. 873-885, (2007)

29. Institute of Transportation Engineers, "Traffic Calming: State of the Practice - Chapter 
6: Legal Authority and Liability," (1999)

30. A. Shirahatti, "Investigation on effect of hump on a vehicle suspension for the comfortable ride," in National Conference on Emerging Trends in Mechanical, (2006)

31. B. Schoettle and M. Sivak, "Public Opinion About Self-Driving Vehicles in China, India, Japan, The U.S., The U.K. and Australia," (2014)

32. J. a. Healey and R. W. Picard, "Detecting Stress During Real-World Driving Tasks Using Physiological Sensors," IEEE Trans. Intell. Transp. Syst., vol. 6, no. 2, pp. 156$166,(2005)$

33. D. J. Fagnant and K. Kockelman, "Preparing a Nation for Autonomous Vehicles: Opportunities, Barriers and Policy Recommendations for Capitalizing on Self-Driven Vehicles," in 93rd Annual Meeting of the Transportation Research Board 2014, (2014) 